\title{
Predictors of Survival in Children With Methymalonic Acidemia With Homocystinuria in Beijing, China: A Prospective Cohort Study
}

\author{
Li Qiliang, Song Wenqi, *Wang Quan, ${ }^{\#}$ Yang Xinying, ${ }^{*}$ Li Jiunei, ${ }^{*}$ Sun Qiang, \$Peng Xiaoxia And Wang \\ PEICHANG \\ From the Departments of Medical Laboratory, ${ }^{*}$ Intensive Care Unit, ${ }^{\#}$ Neurology, ${ }^{\ddagger}$ Nephrology and ${ }^{\$}$ Epidemiology and \\ Biostatistics; Xuanwu Hospital, Beijing Children's Hospital, Capital Medical University, China. \\ Correspondence to: Dr Wang Peichang, Department of Medical Laboratory, Xuanwu Hospital, Capital Medical University, Beijing, 100053, \\ China.peichangwang@yahoo.com \\ Received: February 28, 2014; Initial Review: June 13, 2014; Accepted: November 07,2014.
}

\begin{abstract}
Objective: (i) To determine whether clinical features and biochemical parameters help to predict survival of methylmalonic acidemia with homocystinuria; (ii) To find the cutoff values of biochemical parameters for predicting survival of methylmalonic acidemia with homocystinuria.

Design: A prospective cohort study.

Setting: A pediatric tertiary hospital in Beijing; all patients were followed until death or June 2013.

Subjects: 45 pediatric patients diagnosed with methylmalonic acidemia with homocystinuria between 2006 and 2012 .

Outcome measures: The data of clinical characteristics and pretreatment biochemical parameters were collected. The Cox regression analysis was performed to identify independent risk factors for survival of patients with methylmalonic acidemia and homocystinuria. The best cutoff values for these independent factors were determined by the receiver characteristic curve.
\end{abstract}

Results: Newborn onset $(\mathrm{OR}=6.856,95 \% \mathrm{Cl}=2.241-20.976$, $P=0.001)$, high level of methylmalonic acid in urine $(\mathrm{OR}=1.022$, $95 \% \mathrm{Cl}=1.011-1.033, P<0.001)$, and high level of urea in serum (OR=1.083, 95\% Cl=1.027-1.141, $P=0.003$ ) were independent negative risk factors for survival of patients with methylmalonic acidemia and homocystinuria. The cutoff values of maximum predictive accuracy of methylmalonic acid in urine and urea in serum were respectively $5.41 \mathrm{mmol} / \mathrm{mmol}$ creatinine and 7.80 $\mathrm{mmol} / \mathrm{L}$ by receiver operating characteristic curve analysis.

Conclusion: The patients of methylmalonic acidemia with homocystinuria tend to have an adverse outcome if they have newborn onsets. Elevated urea and urinary methylmalonic acid are predictors of adverse outcomes for the patients. They show similar effect for predicting severe adverse prognosis. The combination of methylmalonic acid in urine concentration and urea in serum concentration provided the most accurate predictive tool.

Key words: Hemocysteine, Methylmalonic acid, Outcome.
$\mathrm{M}$ ethylmalonic acidemia (MMA) is a rare autosomal recessive metabolic disease due to a defect of the mitochondrial enzyme methylmalonyl-CoA mutase (MCM) which converts methylmalonyl-coenzyme A (CoA) into succinyl-CoA, or a defect in the metabolism of 5'deoxyadenosylcobalamin, the cofactor of MCM $[1,2]$. The prevalence of MMA is $1 / 29,000$ in the United States and 1/61,000 in Canada, but still unknown in China [3], though estimated incidence in mainland China is $1 / 26,000$ [1]. According to serum total homocysteine, MMA is divided into isolated MMA and MMA with homocystinuria. MMA with homocystinuria has been reported to common in China [4]. The prognosis of MMA with homocystinuria is poor [5]. We planned this prospective cohort study to validate the performance of clinical characteristics and biochemical parameters to predict survival in chinese children with MMA homocystinuria.

\section{Methods}

This prospective cohort study was conducted from July 2006 to February 2012 at a pediatric tertiary-care hospital in Beijing. Study protocol was approved by Ethics Committee of the hospital and written informed consent was obtained from parents. Urinary organic acids profiles were analyzed by the Gas chromatography-mass spectrometry (GC/MS) in all patients who had the signs/symptoms such as epilepsy, recurrent vomiting, impaired consciousness, recurrent difficulty with feeding, both lower extremities edema, mental retardation or regression. If the level of urinary methylmalonic acid was continuously higher than 100 folds of the normal level $(<0.001 \mathrm{mmol} / \mathrm{mmol}$ creatinine $)$ and a secondary MMA with vitamin $\mathrm{B}_{12}$ deficiency was excluded and the serum homocysteine level was higher than $15 \mathrm{~mol} / \mathrm{L}$ (normal 4-12 mol/L), MMA with homocystinuria was diagnosed [3,6]. All children 


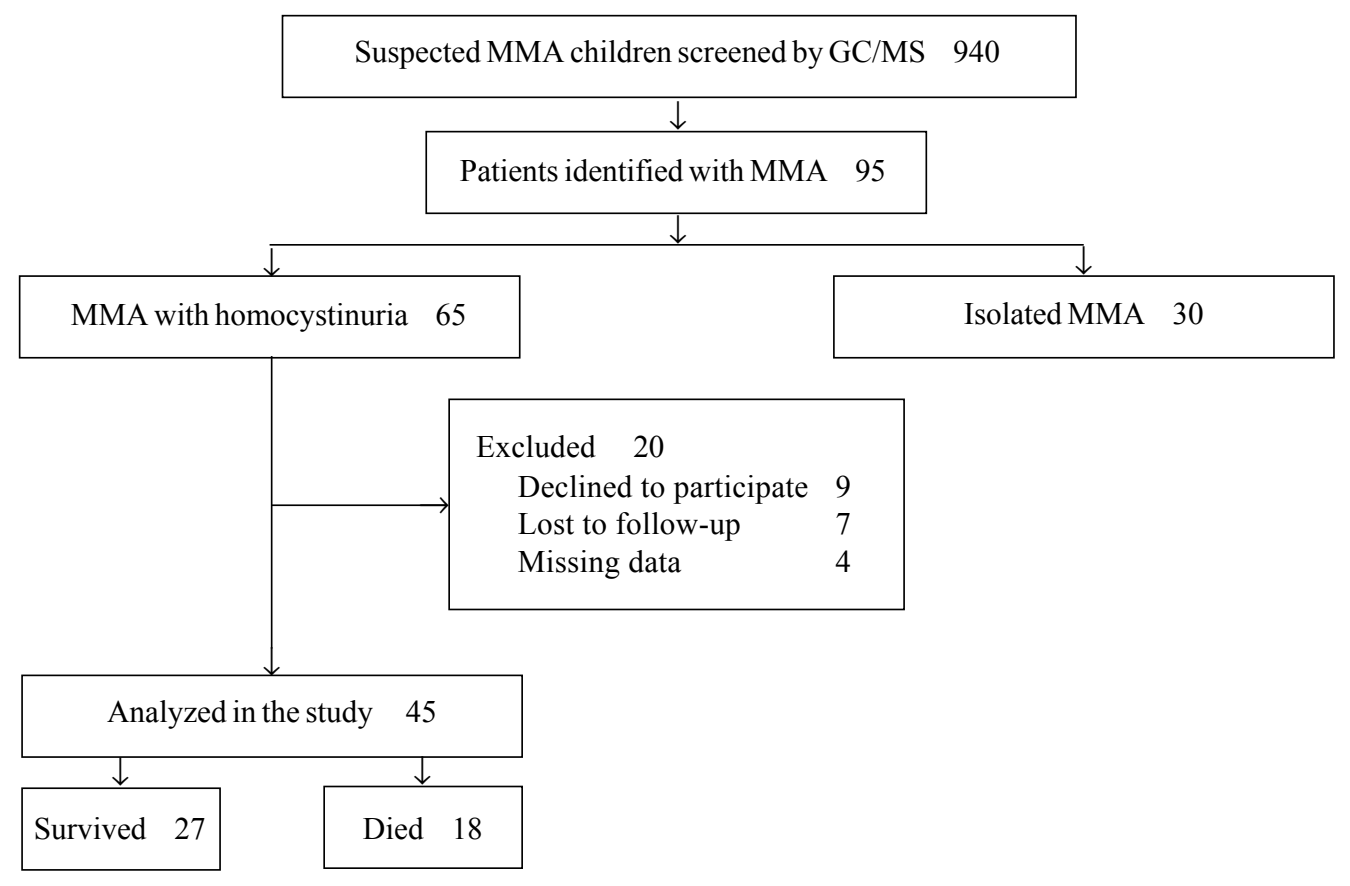

Fig. 1 Study flow.

diagnosed with MMA with homocystinuria were eligible for enrolment in the study (Fig. 1).

Details of history and clinical characteristics were recorded. Pre-treatment biochemical parameters including urinary methylmalonic acid, serum homocysteine, $\mathrm{pH}$, serum creatinine, blood urea nitrogen, uric acid, alanine aminotransferase, aspartate aminotransferase, creatine kinase-MB, lactate dehydrogenase, $\alpha$-hydroxybutyrate dehydrogenase, white blood cell, red blood cell, hemoglobin, occult blood reaction and specific gravity of urine were analyzed. For quality assurance, three levels of internal controls were run with each batch of twenty samples. The intra-assay and interassay variation were less than $3 \%$ and $5 \%$, respectively. The patients received a homogeneous and standardized medical management $[3,4]$; intramuscular injection of vitamin $B_{12} 0.5-1 \mathrm{mg} / \mathrm{d}$ once or twice weekly (or oral mecobalamin tablets $1 \mathrm{mg} / \mathrm{d}$ ), folic acid $2.5-5 \mathrm{mg} / \mathrm{d}$, betaine $500-2000 \mathrm{mg} / \mathrm{d}$, and L-carnitine $250-1000 \mathrm{mg} / \mathrm{d}$. All patients were followed until death or till June 2013 (censor date).

Statistical analysis: All statistical analyses were performed using SPSS version 13.0. Statistical significance was set at $P<0.05$. Categorical variables were compared using the Chi-square test or Fisher exact test, as appropriate. Continuous variables were compared using the Student $t$ test if normally distributed or non-parametric test if non-normally distributed. Kaplan-Meier survival plots were used to display hypothesized relationships and data were compared with the log-rank test. The Cox regression analysis was performed to identify independent risk factors for survival of MMA with homocystinuria. OR and 95\% CI were calculated for risk estimates. For chemical parameters showing a significant association with survival, Receiver operating characteristic (ROC) curve analysis was performed to identify the optimal cutoff points. Areas under the ROC curve, sensitivity, specificity, positive predictive value, and negative predictive value were calculated.

\section{RESUlts}

During the study period, a total of 65 patients diagnosed with MMA and homocystinuria were eligible for enrolment. Among them, 20 were excluded for different reasons (Fig. 1) and 45 patients (28 males) were enrolled in the study (Table I).

At the end of the study, 27 patients were still surviving. The cause of death was renal failure in 10 patients, multiple organ failure in 5 patients, and decompensated metabolic acidosis in 3 patients. Clinical manifestations of 45 patients at diagnosis included epilepsy ( $n=17)$, recurrent vomiting $(n=10)$, impaired consciousness $(n=10)$, recurrent difficulty with feeding $(n=9)$, both lower extremities edema $(n=7)$, mental 
TABLE I Clinical Characteristics OF CHILdREN With Methylmalonic ACIDEMIA AND HomocystinURIa $(N=45)$

\begin{tabular}{lcc}
\hline Characteristic & $\begin{array}{c}\text { Non-survivor } \\
(n=18)\end{array}$ & $\begin{array}{c}\text { Survivor } \\
(n=27)\end{array}$ \\
\hline Male & $9(50)$ & $19(70.4)$ \\
${ }^{*}$ Age at diagnosis, mo & $4.3(0.1-90.2)$ & $8.5(0.1-165)$ \\
${ }^{*}$ Neonatal onset & $6(33.3)$ & $2(7.4)$ \\
Family history & $3(11.1)$ & $1(3.7)$ \\
$\begin{array}{l}\text { \$omiting and/or food refusal } \\
\text { and/or impaired consciousness }\end{array}$ & $15(83.3)$ & $10(37.0)$ \\
\hline
\end{tabular}

All values in $n(\%)$ except *median (range); ${ }^{*} P<0.05$; ${ }^{\$}$ Initial clinical presentation $(P<0.005)$.

retardation or regression $(n=5)$, jaundice $(\mathrm{n}=5)$, anemia $(n=4)$, precordial discomfort $(n=3)$, hypotonia $(n=2)$, tremor $(n=1)$, dysarthria $(n=1)$, ataxia $(n=1)$, coma $(n=1)$ and abnormal posture $(n=1)$. The results are shown in Table I. The occurrence of vomiting, food refusal and impaired consciousness in non-survivors were more than survivors $(P<0.005)$.

Laboratory findings of survivors and non-survivors are compared in Table II. The primary biochemical parameters (e.g. urinary methylmalonic acid, $\mathrm{pH})$ were significantly different between survivors and nonsurvivors. The parameters reflecting kidney (e.g. urea, creatinine and uric acid) and myocardial (e.g. aspartate aminotransferase, creatine kinase-MB, lactate dehydrogenase) injury were significantly higher in nonsurvivors than survivors. In addition, the positive rate of urine occult blood reaction in non-survivors $(12 / 18)$ was significantly higher than survivors $(6 / 27)(P<0.01)$.

The multivariate Cox-proportional hazard model was used to determine independent risk factors of death of patients. Potential risk factors were preliminary screened by Kaplan-Meier survival method and clinical knowledge. The alternative risk factors in the Cox proportional hazard model included with or without newborn onset, with or without the occurrence of vomiting or food refusal or impaired consciousness, concentrations of methylmalonic acid in the urine, the levels of blood urea nitrogen, creatinine, uric acid, aspartate aminotransferase, and creatine kinase-MB. With newborn onset, high levels of methylmalonic acid and urea were independent negative risk factors for survival (Table III). The cut-off values of maximum predictive accuracy of methylmalonic acid in urine and urea in serum, respectively were $5.41 \mathrm{mmol} / \mathrm{mmol}$

TABLE II BASELINE LABORATORY Findings of Non-SURVIVORS AND SuRVIVORs BEFore CLINICAL TREATMENT

\begin{tabular}{lllllr}
\hline Parameters & Unit & Reference range & Non-survivors $(n=18)$ & Survivors $(n=27)$ & P value \\
\hline MMA & mmol/mmol creatinine & $0.000-0.001$ & $7.74(0.11-147.16)$ & $0.34(0.10-20.87)$ & 0.002 \\
$S G$ & - & $1.003-1.030$ & $1.015(1.003-1.035)$ & $1.015(1.005-1.035)$ & $\mathrm{NS}$ \\
$p H$ & $\mathrm{pH}$ units & $7.35-7.45$ & $7.32(7.01-7.42)$ & $7.36(7.07-7.49)$ & 0.01 \\
Ammonia & $\mu \mathrm{mol} / \mathrm{L}$ & $0-54$ & $72(9-700)$ & $55(14-280)$ & $\mathrm{NS}$ \\
Lactate & $\mathrm{mmol} / \mathrm{L}$ & $0.5-2.2$ & $2.15(0.4-4.2)$ & $2.5(0.7-4.8)$ & $\mathrm{NS}$ \\
$H C Y$ & $\mathrm{mmol} / \mathrm{L}$ & $1.9-12.98$ & $75.45(16.20-214.80)$ & $119.10(35.60-219.80)$ & $\mathrm{NS}$ \\
$W B C$ & $10^{9} / \mathrm{L}$ & $4.0-10.0$ & $5.65(1.79-12.60)$ & $6.40(4.80-16.10)$ & $\mathrm{NS}$ \\
$R B C$ & $10^{12} / \mathrm{L}$ & $3.5-5.5$ & $3.18(1.66-5.43)$ & $3.31(2.12-4.56)$ & $\mathrm{NS}$ \\
$H G B$ & $\mathrm{~g} / \mathrm{L}$ & $110.0-160.0$ & $102.50(60.0-174.0)$ & $108.00(73.0-137.0)$ & $\mathrm{NS}$ \\
$B U N$ & $\mathrm{mmol} / \mathrm{L}$ & $1.7-7.1$ & $8.64(2.11-16.64)$ & $3.21(1.69-10.20)$ & 0.001 \\
$C R$ & $\mu \mathrm{mol} / \mathrm{L}$ & $27.0-130.0$ & $77.55(22.10-421.00)$ & $43.20(18.90-83.80)$ & 0.012 \\
$U A$ & $\mu \mathrm{mol} / \mathrm{L}$ & $119.0 \sim 416.0$ & $455(213.0-1194.8)$ & $255(134.0-1113.4)$ & 0.006 \\
$A L T$ & $\mathrm{U} / \mathrm{L}$ & $5.0-40.0$ & $32.35(12.0-54.0)$ & $21.00(8.2-60.6)$ & $\mathrm{NS}$ \\
$A S T$ & $\mathrm{U} / \mathrm{L}$ & $5.0-40.0$ & $53.65(28.40-157.00)$ & $31.00(17.50-163.70)$ & 0.001 \\
$C K-M B$ & $\mathrm{U} / \mathrm{L}$ & $0.0-25.0$ & $21.85(10.0-225.0)$ & $17.50(10.0-55.3)$ & 0.029 \\
$L D H$ & $\mathrm{U} / \mathrm{L}$ & $50.0-240.0$ & $314.00(60.0-1685.0)$ & $244.00(134.0-3347.0)$ & 0.034 \\
$H B D H$ & $\mathrm{U} / \mathrm{L}$ & $80.0-220.0$ & $255.00(89.0-1335.0)$ & $195.00(120.0-2886.0)$ & $\mathrm{NS}$ \\
\hline
\end{tabular}

Values in median (range) NS - not significant; MMA - Methylmalonic acid; SG - specific gravity of urine; $p H$ - arterial blood pH; HCY Homocysteine; WBC - White blood cells; RBC-Red blood cells; HGB - Hemoglobin; BUN-Blood urea nitrogen; CR - Creatinine; UA - Uric acid ; ALT - Alanine aminotransferase; AST - Aspartate aminotransferase; CK-MB - Creatine kinase-MB; LDH - Lactate dehydrogenase; HBDH - $\alpha$ hydroxybutyrate dehydrogenase. 
TABLE III Prognostic Factors Associated With Death IN Children with Methylmalonic Acidemia with HOMOCYSTINURIA.

\begin{tabular}{lll}
\hline Variables & Pvalue & OR $(95 \% C I)$ \\
\hline With newborn onset & 0.001 & $6.856(2.241,20.976)$ \\
MMA in urine & 0.000 & $1.022(1.011,1.033)$ \\
BUN in serum & 0.003 & $1.083(1.027,1.141)$ \\
\hline
\end{tabular}

CI- confidence interval; OR-odd ratio; MMA-Methylmalonic acid; BUN-Blood urea nitrogen; significance level of 0.05 or less.

creatinine and $7.80 \mathrm{mmol} / \mathrm{L}$ by Receiver operating characteristic curve analysis. The performance of methylmalonic acid in urine as an individual factor for identifying patients at a high risk of death was similar with urea in serum (Table IV). The combination of methylmalonic acid in urine and urea in serum provided the most accurate predictive tool (e.g. increased sensitivity without decreased specificity).

\section{Discussion}

Some retrospective studies reported that the patients with methylmalonic acidemia had different long term outcomes [4,7]. In order to find out predictors for survival of MMA with homocystinuria, we analyzed clinical features and biochemical parameters of 45 Chinese pediatric patients and assessed the predictive ability.

Among the clinical manifestations, recurrent vomiting, food refusal, and impaired consciousness were reported by Zwickler, et al. [8] as life-threatening alarming symptoms of patients with MMA. Although we also found the more occurrences of recurrent vomiting, food refusal, and impaired consciousness in nonsurvivors, the three symptoms were not individual risk factors for death of the patients. The possible cause of inconsistency in the two studies is probable due to different populations. Zwickler, et al. [8] selected patients diagnosed with isolated MMA as research subjects. However, our research subjects were children diagnosed as MMA with homocystinuria. Because the pathogenic mechanisms between isolated methylmalonic academia and methylmalonic academia with homocystinuria are different [4,9], the alarming symptoms may have different predictable values.

Newborn onset was found to be a major clinical feature to predict negative outcome of MMA with homocystinuria, possibly because these patients have more severe complications e.g. decompensated metabolic acidosis, renal failure and multiple organ failure, or the diagnosis is often missed as clinical symptoms during neonatal period are not apparent or specific [10]. Thus, irreversible damage such as organ failure had already occurred by the time these patients were seen at our center. It is expected that routine newborn screening can reduce morbidity and mortality by early diagnosis and early treatment $[11,12]$.

In this study, some biochemical parameters indicated more metabolic decompensation and more severe renal injury and myocardial injury in non-survivors than survivors. Hörster, et al. [15] reported that high concentrations of MMA in urine are a known risk factor for the development of chronic kidney disease. Moreover, homocysteine can induce myocardial injury by promotion of endothelial dysfunction, formation of thromboxane A2, enhancement of platelet aggregation, reduction in the protective effect of nitric oxide, and the procoagulant effects [4-17]. The disturbance of the tricarboxylic acid cycle and respiratory chain may also be involved in the pathogenesis [18]. In addition, mitochondrial dysfunction, oxidative stress and disturbances in mitochondrial DNA equilibrium may be associated with organ injury [19-21].

We found that elevated urinary methylmalonic acid was one biochemical predictor of adverse outcomes for MMA with homocystinuria. Ledley, et al. [22] found that children who had low levels of methylmalonic acid in blood and urine had good outcomes [22]. However, there is no report about the cut-off value of urinary methylmalonic acid to predict long term outcomes of MMA with homocystinuria. In our study, the level of urinary methylmalonic acid $>5.41 \mathrm{mmol} / \mathrm{mmol}$

TABLE IV Prognostic Factors for Poor Outcome in Children With Methylmalonic Acidemia ANd Homocystinuria

\begin{tabular}{llcccc}
\hline Finding & AUC & Sensitivity (\%) & Specificity(\%) & PPV (\%) & NPV(\%) \\
\hline Urinary MMA $>5.41 \mathrm{mmol} / \mathrm{mmol}$ creatinine & $0.778 \pm 0.071$ & 72.2 & 81.5 & 81.3 & 82.8 \\
$\begin{array}{l}\text { Urea }>7.80 \mathrm{mmol} / \mathrm{L} \\
\text { Urinary MMA }>5.41 \mathrm{mmol} / \mathrm{mmol} \text { creatinine }\end{array}$ & $0.787 \pm 0.070$ & 55.6 & 89.9 & 76.9 & 75.0 \\
$\quad$ or Urea $>7.80 \mathrm{mmol} / \mathrm{L}$ & $0.901 \pm 0.035$ & 77.8 & 96.3 & 93.3 & 86.7 \\
\hline
\end{tabular}

AUC-area under the curve; PPV-positive predictive value; $N P V$ - negative predictive value; MMA-Methylmalonic acid. 
creatinine (5410 folds of normal level) showed high specificity for identifying patients at increased risk for death. However, as an independent risk factor, the sensitivity of urinary methylmalonic acid was limited.

Blood urea nitrogen was the other biochemical predictor of adverse outcomes for MMA with homocystinuria. Our study found that renal failure was one of the main causes of the death. Blood urea nitrogen, an important biochemical parameter reflecting renal function, showed prediction effect for death of patients. However, it is very interesting that there is a discrepancy of blood urea nitrogen and serum creatinine with different prognostic value for outcome. The rise of blood urea nitrogen had prognostic significance, but serum creatinine had not. In order to find out whether the rise of blood urea nitrogen resulted from the prerenal azotemia induced by reduced blood volume, we used the parameter of specific gravity of urine to evaluate the water balance in children. The results showed that specific gravity of urine was not significantly different between the two groups. In addition, the positive rate of urine occult blood reaction in non-survivors was significantly higher than survivors. So we think that the rise of blood urea nitrogen may result from postrenal azotemia. We found that the most patients were in poor nutrition state because of vomiting and food refusal, leading to a loss of muscle mass thereby reducing creatinine synthesis. Therefore, the level of serum creatinine could not reflect accurately the renal function. The blood urea nitrogen level with the greatest prognostic ability was $7.80 \mathrm{mmol} / \mathrm{L}$, which had a similar specificity with urinary methylmalonic acid $>5.41$ $\mathrm{mmol} / \mathrm{mmol}$ creatinine for predicting severe adverse prognosis of MMA with homocystinuria. In addition, combination of elevated urinary methylmalonic acid or elevated blood urea nitrogen provided better predictive ability by increasing sensitivity compared with each variable considered separately.

Relevant pathogenic genes (MMACHC, MMADHC, $L M B R D 1$ ) were not detected in the present study due to lack of facilities. Thus, we could not get the information of different gene and different mutations risk assessment for survival of MMA with homocystinuria.

In conclusion, with newborn onset, higher levels of urinary methylmalonic acid and blood urea nitrogen increase risk for death of MMA with homocystinuria. Further studies are needed to validate performance of pathogenic genes to predict survival of the patients with MMA and homocystinuria.

Contributors: LQ: participated in the design, carried out the experimental work, collection and interpretation of the data and drafted the manuscript; WQ, YX, LJ, SQ: participated in the design and coordination of experimental work, and acquisition of clinical data; PX: participated in the study design, data collection, analysis of data and preparation of the manuscript; WP, SW: carried out the study design, the analysis and interpretation of data. All authors approved the manuscript for submission.

Funding: Special Program for Capital Clinical Research of the Beijing Municipal Commission of Science and Technology, China (Grant No. Z121107005112008).

Competing interests: None stated.

\section{REFERENCES}

1. Tu WJ. Methylmalonic acidemia in mainland China. Ann Nutr Metab. 2011;58:281.

2. Carrillo-Carrasco N, Chandler RJ, Venditti CP. Combined methylmalonic acidemia and homocystinuria, cblC type. I. Clinical presentations, diagnosis and management. J Inherit Metab Dis. 2012;35:91-102.

3. Ma X, Zhang Y, Yang Y, Liu X, Yang Z, Bao X, et al. Epilepsy in children with methylmalonic acidemia: electroclinical features and prognosis. Brain Dev. 2011;33:790-5.

4. Huang Z, Han LS, Ye J, Qiu WJ, Zhang HW, Gao XL, et al. Outcomes of patients with combined methylmalonic acidemia and homocystinuria after treatment. Zhonghua Er Ke Za Zhi. 2013;51:194-8.

5. Rosenblatt DS, Aspler AL, Shevell MI, Pletcher BA, Fenton WA, Seashore MR. Clinical heterogeneity and prognosis in combined methylmalonic aciduria and homocystinuria (cblC). J Inherit Metab Dis. 1997;20: 528-38.

6. Zhang Y, Song JQ, Liu P, Yan R, Dong JH, Yang YL, et al. Clinical studies on fifty-seven Chinese patients with combined methylmalonic aciduria and homocysteinemia. Zhonghua Er Ke Za Zhi. 2007;45:513-7.

7. Cosson MA, Benoist JF, Touati G, Déchaux M, Royer N, Grandin L, et al. Long-term outcome in methylmalonic aciduria: a series of 30 French patients. Mol Genet Metab. 2009;97:172-8.

8. Zwickler T, Haege G, Riderer A, Hörster F, Hoffmann $\mathrm{GF}$, Burgard $\mathrm{P}$, et al. Metabolic decompensation in methylmalonic aciduria: which biochemical parameters are discriminative? J Inherit Metab Dis. 2012; 35: 797-806.

9. Vatanavicharn N, Champattanachai V, Liammongkolkul S, Sawangareetrakul P, Keeratichamroen S, Ketudat Cairns JR, et al. Clinical and molecular findings in Thai patients with isolated methylmalonic acidemia. Mol Genet Metab. 2012;106:424-9.

10. Tu W, He J, Dai F, Wang X, Li Y. Impact of inborn errors of metabolism on admission in a neonatal intensive care unit - a prospective cohort study. Indian $\mathrm{J}$ Pediatr. 2012;79:494-500.

11. Nagaraja D, Mamatha SN, De T, Christopher R. Screening for inborn errors of metabolism using automated electrospray tandem mass spectrometry: study in high-risk Indian population. Clin Biochem. 2010;43:581-8. 
12. Sun W, Wang Y, Yang Y, Wang J, Cao Y, Luo F, et al. The screening of inborn errors of metabolism in sick Chinese infants by tandem mass spectrometry and gas chromatography/mass spectrometry. Clin Chim Acta. 2011;412:1270-4.

13. Hörster F, Baumgartner MR, Viardot C, Suormala T, Burgard $\mathrm{P}$, Fowler $\mathrm{B}$, et al. Long-term outcome in methylmalonic acidurias is influenced by the underlying defect (mut0, mut-, cblA, cblB). Pediatr Res. 2007; 62:225-30

14. Durand P, Lussier-Cacan S, Blache D. Acute methionine load-induced hyperhomocysteinemia enhances platelet aggregation, thromboxane biosynthesis, and macrophagederived tissue factor activity in rats. FASEB J. 1997; 11:1157-68.

15. Woo KS, Sanderson JE, Sun YY, Chook P, Cheung AS, Chan LT, et al. Hyperhomocyst(e)inemia is a risk factor for arterial endothelial dysfunction in humans. Circulation. 2000;101:E116.

16. Stamler JS, Osborne JA, Jaraki O, Rabbani LE, Mullins M, Singel D. Adverse vascular effects of homocysteine are modulated by endothelium-derived relaxing factor and related oxides of nitrogen. J Clin Invest. 1993; 91:308-18.
17. Mayer EL, Jacobsen DW, Robinson K. Homocysteine and coronary atherosclerosis. J Am Coll Cardiol. 1996;27:517-27.

18. Morath MA, Okun JG, Müller IB, Sauer SW, Hörster F, Hoffmann GF, et al. Neurodegeneration and chronic renal failure in methylmalonic aciduria - a pathophysiological approach. J Inherit Metab Dis. 2008; 31:35-43.

19. de Keyzer Y, Valayannopoulos V, Benoist JF, Batteux F, Lacaille F, Hubert L, et al. Multiple OXPHOS deficiency in the liver, kidney, heart, and skeletal muscle of patients with methylmalonic aciduria and propionic aciduria. Pediatr Res. 2009;66:91-5.

20. Kölker S, Sauer SW, Surtees RA, Leonard JV. The aetiology of neurological complications of organic acidaemias - a role for the blood-brain barrier. J Inherit Metab Dis. 2006;29:701-4.

21. Kölker S, Burgard P, Sauer SW, Okun JG. Current concepts in organic acidurias: understanding intra- and extracerebral disease manifestation. J Inherit Metab Dis. 2013;36:635-44.

22. Ledley FD, Levy HL, Shih VE, Benjamin R, Mahoney MJ. Benign methylmalonic aciduria. N Engl J Med. 1984;311:1015-8. 